\title{
Erratum: Lattice Dynamics of EuO: Evidence for Giant Spin-Phonon Coupling [Phys. Rev. Lett. 116, 185501 (2016)]
}

\author{
R. Pradip, P. Piekarz, A. Bosak, D. G. Merkel, O. Waller, A. Seiler, A. I. Chumakov, R. Rüffer, \\ A. M. Oleś, K. Parlinski, M. Krisch, T. Baumbach, and S. Stankov \\ (Received 24 July 2017; published 18 August 2017)
}

DOI: 10.1103/PhysRevLett.119.079903

We have found a mistake in the value of the normalized mean force constant $K$ determined from the phonon DOS. The correct value reported on p. 185501-4, left column, line 6 from bottom is $K=6.3 \mathrm{eV} / \AA^{2}$. This results in a spin-phonon coupling constant $\alpha \approx 16$ and the corresponding $\alpha_{s-f} \approx 12$. These values are even higher from the ones originally reported in the Letter, thus confirming the giant spin-phonon interaction in EuO. Figure S6(c) from the Supplemental Material has been updated and the given values of the normalized mean force constant $K$ are correct. 